\title{
Dynamics during outburst
}

\section{VLTI observations of the young eruptive star V1647 Orionis during its 2003-2006 outburst ${ }^{\star}$}

\author{
L. Mosoni ${ }^{1,2}$, N. Sipos ${ }^{1,3}$, P. Ábrahám ${ }^{1}$, A. Moór ${ }^{1}$, Á. Kóspál ${ }^{4,5, \star \star}$, Th. Henning ${ }^{2}$, A. Juhász ${ }^{2,4}$, M. Kun ${ }^{1}$, Ch. Leinert² \\ S. P. Quanz ${ }^{6}$, Th. Ratzka ${ }^{7}$, A. A. Schegerer ${ }^{2}$, R. van Boekel ${ }^{2}$, and S. Wolf ${ }^{2,8}$
}

1 Konkoly Thege Miklós Astronomical Institute, Research Centre for Astronomy and Earth Sciences,
Hungarian Academy of Sciences, PO Box 67, 1525 Budapest, Hungary
e-mail: mosoni@konkoly.hu
2 Max Planck Institute for Astronomy, Königstuhl 17, 69117 Heidelberg, Germany
3 Universiät Göttingen, Institut für Astrophysik, Friedrich-Hund-Platz 1, 37077 Göttingen, Germany
4 Leiden Observatory, Leiden University, PO Box 9513, 2300 RA Leiden, The Netherlands
5 Research and Scientific Support Department, European Space Agency (ESA-ESTEC, SRE-SA), PO Box 299, 2200 AG Noordwijk,
The Netherlands
6 Institute for Astronomy, ETH Zürich, Wolfgang-Pauli-Strasse 27, 8093 Zürich, Switzerland
7 Universitäts-Sternwarte München, Ludwig-Maximilians-Universität, Scheinerstr. 1, 81679 München, Germany
8 Christian-Albrechts-Universität zu Kiel, Institut für Theoretische Physik und Astrophysik, Leibnizstrasse 15, 24098 Kiel, Germany

Received 3 January 2012 / Accepted 8 January 2013

\begin{abstract}
Context. It is hypothesized that low-mass young stellar objects undergo eruptive phases during their early evolution. These eruptions are thought to be caused by highly increased mass accretion from the disk onto the star, and therefore play an important role in the early evolution of Sun-like stars, of their circumstellar disks (structure, dust composition), and in the formation of their planetary systems. The outburst of V1647 Ori between 2003 and 2006 offered a rare opportunity to investigate such an accretion event.

Aims. By means of our interferometry observing campaign during this outburst, supplemented by other observations, we investigate the temporal evolution of the inner circumstellar structure of V1647 Ori, the region where Earth-like planets could be born. We also study the role of the changing extinction in the brightening of the object and separate it from the accretional brightening.

Methods. We observed V1647 Ori with MIDI on the VLTI at two epochs in this outburst. First, during the slowly fading plateau phase (2005 March) and second, just before the rapid fading of the object (2005 September), which ended the outburst. We used the radiative transfer code MC3D to fit the interferometry data and the spectral energy distributions from five different epochs at different stages of the outburst. The comparison of these models allowed us to trace structural changes in the system on AU-scales. We also considered qualitative alternatives for the interpretation of our data.

Results. We found that the disk and the envelope are similar to those of non-eruptive young stars and that the accretion rate varied during the outburst. We also found evidence for the increase of the inner radii of the circumstellar disk and envelope at the beginning of the outburst. Furthermore, the change of the interferometric visibilities indicates structural changes in the circumstellar material. We test a few scenarios to interpret these data. We also speculate that the changes are caused by the fading of the central source, which is not immediately followed by the fading of the outer regions.

Conclusions. We found that most of our results fit in the canonical picture of young eruptive stars. Our study provided dynamical information from the regions of the innermost few AU of the system: changes of the inner radii of the disk and envelope. However, if the delay in the fading of the disk is responsible for the changes seen in the MIDI data, the effect should be confirmed by dynamical modeling.
\end{abstract}

Key words. stars: formation - circumstellar matter - stars: individual: V1647 Ori - infrared: stars - techniques: interferometric

\section{Introduction}

The origin of the matter of low-mass stars is interstellar gas and dust, which fall onto the protostar through a circumstellar accretion disk. It is becoming increasingly evident that the

\footnotetext{
* Based on observations made with ESO telescopes at the Paranal Observatory under program IDs 274.C-5026 and 076.C-0736. In addition, this work is based in part on archival data obtained with the Spitzer Space Telescope, which is operated by the Jet Propulsion Laboratory, California Institute of Technology under a contract with NASA.

$\star \star$ ESA fellow.
}

accumulation of stellar material is an episodic process: the typically low accretion rate suddenly increases for limited periods, delivering a significant amount of mass onto the stellar surface (e.g., Evans et al. 2009). Many young stars exhibit temporal brightenings possibly caused by fluctuating accretion (Herbst et al. 1994; Sicilia-Aguilar et al. 2008). The eruptions of the FU Orionis- and EX Lupi-type classes of variable stars (FUors and EXors) may represent the most intense bursts (e.g., Hartmann \& Kenyon 1996, hereafter HK96; Herbig 2007). Such outbursts are characterized by an optical brightening of 2-6 mag. FUor eruptions can last from some years to several 
decades, while EXor-outbursts are usually considered as shorter counterparts of those of FUors (i.e., some months long). A number of possible scenarios for the triggering mechanism of the outburst have been proposed: (a) interactions of binary or multiple systems where tidal forces disturb the circumstellar disk (Bonnell \& Bastien 1992); (b) thermal instabilities in the disk (Bell et al. 1995); (c) planet-disk interactions, where thermal instabilities in the disk are caused by the presence of a massive planet (Lodato \& Clarke 2004); (d) gravitational instabilities in the disk (Vorobyov \& Basu 2006; Zhu et al. 2009); or (e) interaction between the disk and the magnetic field (D'Angelo \& Spruit 2010). The difference between the physics of FUor and EXor-outbursts is unclear. Although currently only about $20 \mathrm{ob}-$ jects are classified as eruptive young stellar objects (e.g., HK96; Sandell \& Weintraub 2001; Ábrahám et al. 2004), on the basis of statistical arguments it is believed that most low-mass premain sequence stars undergo FUor and/or EXor phases. Thus understanding the physics of the outbursts will shed light on fundamental processes of the early evolution of Sun-like stars and their circumstellar disks (structure, dust composition) and in turn on the changes of the conditions in the disks that govern the formation of planetary systems. The importance of the outburst phase in the early disk evolution was demonstrated for instance by Ábrahám et al. (2009), who discovered episodic crystallization of silicate grains on the disk surface due to the increased luminosity during the 2008 outburst of EX Lup, resulting in material that forms the building blocks of comets and planets.

One of the best-documented and most-studied outbursts in the history of eruptive stars is that of V1647 Ori in 2003-2006 (see the references collected by Aspin \& Reipurth 2009). The low-mass central object $\left(M_{\text {star }}=0.8 M_{\odot}, T_{\text {star }}=3800 \mathrm{~K}\right.$, $R_{\text {star }}=3.25 R_{\odot}{ }^{1}$; Aspin et al. 2008), deeply embedded in the dark cloud LDN 1630, brightened by $\sim 4.5 \mathrm{mag}$ in $I_{\mathrm{C}}$ during three months (Briceño et al. 2004) until it reached a peak brightness in early 2004. It illuminated a new conical reflection nebulosity, the McNeil Nebula (McNeil 2004). Subsequently, the object slowly faded during about 20 months. Between September-October 2005 and early 2006 the object rapidly faded to its quiescent brightness (Acosta-Pulido et al. 2007, hereafter AP07). Variation of the near-infrared colors along the reddening path suggests that the brightening was partly caused by a temporal removal of an extinction slab (e.g., Reipurth \& Aspin 2004). Optical and near-infrared spectroscopic observations (e.g., Reipurth \& Aspin 2004) showed characteristic spectral features attributed to accretion ( $\mathrm{Br} \gamma$ emission) and significant mass-loss (strong $\mathrm{H} \alpha$ emission with a PCyg profile). The variation of the accretion rate followed that of the optical brightness of the object, i.e., increased rapidly at the beginning of outburst, but decreased later on (AP07). The variation of the X-ray emission, which must originate from accretion processes, also follows the optical light curve, which supports the accretion scenario (Teets et al. 2011).

Despite the numerous studies (see references in Aspin \& Reipurth 2009), the circumstellar structure of V1647 Ori, and especially its variations, have not been investigated in detail yet ${ }^{2}$. With the aim of studying the circumstellar disk and envelope of V1647 Ori and the dynamical processes during the eruption, we fitted the spectral energy distribution (SED) of the source at several epochs. In order to achieve a better characterization

\footnotetext{
The radius was recalculated from the post-outburst luminosity considering a distance of $d=400 \mathrm{pc}$ (Anthony-Twarog 1982).

2 The study of Muzerolle et al. (2005) was confined to using simple accretion-disk/remnant-envelope models for data of a single epoch.
}

of V1647 Ori, we initiated multi-epoch observations with the VLTI/MIDI mid-infrared interferometer. Spatial information on high angular scales was collected during two epochs. The first dataset from 2005 March was published in our preceding paper (Ábrahám et al. 2006, hereafter Paper I). Now we analyze these data together with the interferometric data from 2005 September, and with spectral energy distributions at different phases of the outburst. We performed detailed modeling of the circumstellar environment at each epoch to decide whether the observed temporal changes are related to the varying illumination of the disk by its central region or are caused by a change of the disk structure. The results can be directly compared with those on another young eruptive star, PV Cep (Kun et al. 2011), whose observed flux variations are partly explained by variable extinction in the innermost part of the system, due to evaporation and recondensation of dust grains caused by the changing amounts of energy released during the outburst. Variations of the model parameters, together with their timescales, give insight into the dynamics of the outburst of the low-mass pre-main sequence star V1647 Ori.

In Sect. 2 we describe the MIDI, Spitzer, and ground-based photometric observations and their data reduction. In Sect. 3 we present the results: the interferometric data, optical and infrared light curves, and compilations of the SED. In Sect. 4 we describe the radiative transfer models and discuss the temporal variation of physical parameters. Section 5 contains our conclusions.

\section{Observations and data reduction}

\subsection{VLTI/MIDI}

During the 2003-2006 outburst, V1647 Ori was successfully observed twice with MIDI on the VLTI (Leinert et al. 2003): on 2005 March 2 and 2005 September 19. The projected baseline lengths were 56 and $62 \mathrm{~m}$ with position angles of 111 and $108^{\circ}$. These baselines allow one to marginally resolve structures of objects with sizes of the mid-infrared-emitting regions of $\geq 3$ mas, which corresponds to $\geq 1.2 \mathrm{AU}$ at the distance of V1647 Ori. The two observing runs were carried out on the UT3-UT4 baseline of the VLTI. V1647 Ori was observed together with the spectrophotometric calibrator HD 37160. Owing to the optical faintness of the object and the lack of an adequate guide star, MACAO (the adaptive optics system of the VLTI for the Unit Telescopes) could not support the observations. At the time of our first observations, the seeing was $\approx 1^{\prime \prime}$. During the observations in September, it was better, 0.6-0.7". In this latter case, the atmospheric effects on the beam-overlap were less severe.

The obtained sets of data consist of acquisition images with the N8.7 filter, $8-13 \mu \mathrm{m}$ low-resolution $(R=30)$ spectra, and interferometric measurements. In the data reduction we followed the general processing scheme as described in previous papers (e.g., Leinert et al. 2004 or Ratzka et al. 2007). MIDI data can be reduced in two independent ways: with the MIDI Interactive Analysis (MIA) package, which uses the power spectrum method, and the Expert Work Station (EWS) package, which is based on a coherent, linear averaging method $\left(\right.$ Chesneau 2007) ${ }^{3}$. Since V1647 Ori is not a bright object, we set up the observations in such a way that the scanning optical path delay (OPD) passes through the true zero OPD. In this case MIA should work more reliably than EWS. Nevertheless, we reduced the data with the 1.6 version of both softwares and found that the results of the two agree well. We also tested the results by using different mask widths, but found only a few percent effect on the

\footnotetext{
3 The EWS+MIA package can be obtained from http://www. strw . leidenuniv.nl/ nevec/MIDI/
} 
Table 1. Summary of observations of V1647 Ori.

\begin{tabular}{|c|c|c|c|c|c|}
\hline Date & Instrument & Band/Wavelength & Magnitude/flux [Jy] & Notes & References \\
\hline 2007 Jan. 20 & IAC-80 & $I_{\mathrm{J}}$ & $19.24 \pm 0.06 \mathrm{mag}$ & & \\
\hline 2007 Oct. 28 & IAC- 80 & $I_{\mathrm{J}}$ & $19.26 \pm 0.10 \mathrm{mag}$ & & \\
\hline 2008 Feb. 6 & $\mathrm{RCC}$ & $I_{\mathrm{C}}$ & $19.54 \pm 0.06 \mathrm{mag}$ & & \\
\hline 2007 Jan. 20 & TCS & $J$ & $14.60 \pm 0.06 \mathrm{mag}$ & & \\
\hline 2007 Oct. 22 & TCS & $J$ & $14.58 \pm 0.06 \mathrm{mag}$ & & \\
\hline 2007 Oct. 28 & TCS & $J$ & $14.44 \pm 0.05 \mathrm{mag}$ & & \\
\hline 2007 Jan. 20 & TCS & $\bar{H}$ & $11.89 \pm 0.06 \mathrm{mag}$ & & \\
\hline 2007 Oct. 22 & TCS & $H$ & $11.96 \pm 0.12 \mathrm{mag}$ & & \\
\hline 2007 Oct. 28 & TCS & $H$ & $11.70 \pm 0.05 \mathrm{mag}$ & & \\
\hline 2007 Jan. 20 & TCS & $K_{\mathrm{s}}$ & $10.01 \pm 0.04 \mathrm{mag}$ & & \\
\hline 2007 Oct. 22 & TCS & $K_{\mathrm{s}}$ & $9.86 \pm 0.04 \mathrm{mag}$ & & \\
\hline 2007 Oct. 28 & TCS & $K_{\mathrm{s}}$ & $9.77 \pm 0.06 \mathrm{mag}$ & & \\
\hline 2004 Mar. 28 & Spitzer/IRAC & $3.6 \mu \mathrm{m}$ & $1.883 \pm 0.058$ & 9463808 & \\
\hline 2004 Oct. 27 & Spitzer/IRAC & $3.6 \mu \mathrm{m}$ & $0.914 \pm 0.029$ & 12260864 & \\
\hline 2005 Feb. 25 & Spitzer/IRAC & $3.6 \mu \mathrm{m}$ & $0.945 \pm 0.034$ & 11570176 & \\
\hline 2005 Mar. 25 & Spitzer/IRAC & $3.6 \mu \mathrm{m}$ & $1.117 \pm 0.029$ & 11576320 & \\
\hline 2004 Mar. 28 & Spitzer/IRAC & $4.5 \mu \mathrm{m}$ & $2.864 \pm 0.087$ & 9463808 & \\
\hline 2004 Oct. 27 & Spitzer/IRAC & $4.5 \mu \mathrm{m}$ & $1.353 \pm 0.044$ & 12260864 & \\
\hline 2005 Feb. 25 & Spitzer/IRAC & $4.5 \mu \mathrm{m}$ & $1.443 \pm 0.051$ & 11570176 & \\
\hline 2005 Mar. 25 & Spitzer/IRAC & $4.5 \mu \mathrm{m}$ & $1.667 \pm 0.042$ & 11576320 & \\
\hline 2004 Mar. 28 & Spitzer/IRAC & $5.8 \mu \mathrm{m}$ & $3.713 \pm 0.111$ & 9463808 & \\
\hline 2004 Oct. 27 & Spitzer/IRAC & $5.8 \mu \mathrm{m}$ & $1.716 \pm 0.055$ & 12260864 & \\
\hline 2005 Feb. 25 & Spitzer/IRAC & $5.8 \mu \mathrm{m}$ & $1.839 \pm 0.062$ & 11570176 & \\
\hline 2005 Mar. 25 & Spitzer/IRAC & $5.8 \mu \mathrm{m}$ & $2.062 \pm 0.052$ & 11576320 & \\
\hline 2004 Mar. 28 & Spitzer/IRAC & $8.0 \mu \mathrm{m}$ & $5.721 \pm 0.172$ & 9463808 & \\
\hline 2004 Oct. 21 & Spitzer/IRS ( $(\star)$ & $8.0 \mu \mathrm{m}$ & $3.71 \pm 0.19$ & 12261120 & \\
\hline 2004 Oct. 27 & Spitzer/IRAC & $8.0 \mu \mathrm{m}$ & $2.745 \pm 0.090$ & 12260864 & \\
\hline 2005 Feb. 25 & Spitzer/IRAC & $8.0 \mu \mathrm{m}$ & $3.008 \pm 0.097$ & 11570176 & \\
\hline 2005 Mar. 2 & VLTI/MIDI & $8.7 \mu \mathrm{m}$ & $3.5 \pm 0.5$ & & Ábrahám et al. (2006) \\
\hline 2005 Mar. 11 & Spitzer/IRS ( $(\star)$ & $8.0 \mu \mathrm{m}$ & $2.32 \pm 0.12$ & 11569920 & \\
\hline 2005 Mar. 24 & Spitzer/IRS ( $(\star)$ & $8.0 \mu \mathrm{m}$ & $2.86 \pm 0.14$ & 12644096 & \\
\hline 2005 Mar. 25 & Spitzer/IRAC & $8.0 \mu \mathrm{m}$ & $3.217 \pm 0.083$ & 11576320 & \\
\hline 2005 Sep. 19 & VLTI/MIDI & $8.7 \mu \mathrm{m}$ & $2.0 \pm 0.5$ & & \\
\hline 2004 Oct. 21 & Spitzer/IRS & $4-34 \mu \mathrm{m}$ & & 12261120 & Quanz et al. (2007) \\
\hline 2005 Mar. 2 & VLTI/MIDI & $8-13 \mu \mathrm{m}$ & & $56 \mathrm{~m} / 111^{\circ}$ & Ábrahám et al. (2006) \\
\hline 2005 Mar. 11 & Spitzer/IRS & $4-34 \mu \mathrm{m}$ & & 11569920 & Quanz et al. (2007) \\
\hline 2005 Mar. 24 & Spitzer/IRS & $4-34 \mu \mathrm{m}$ & & 12644096 & Quanz et al. (2007) \\
\hline 2005 Sep. 19 & VLTI/MIDI & $8-13 \mu \mathrm{m}$ & & $62 \mathrm{~m} / 108^{\circ}$ & \\
\hline 2004 Mar. 15 & Spitzer/MIPS & $24 \mu \mathrm{m}$ & $18.2 \pm 2.8$ & 4320256 & Muzerolle et al. (2004) \\
\hline 2004 Oct. 14 & Spitzer/MIPS & $24 \mu \mathrm{m}$ & $16.5 \pm 2.5$ & 12260352 & \\
\hline 2004 Oct. 21 & Spitzer/IRS ( $(\star)$ & $24 \mu \mathrm{m}$ & $13.68 \pm 0.71$ & 12261120 & \\
\hline 2005 Mar. 11 & Spitzer/IRS ( $(\star)$ & $24 \mu \mathrm{m}$ & $8.65 \pm 0.45$ & 11569920 & \\
\hline 2005 Mar. 24 & Spitzer/IRS ( $(\star)$ & $24 \mu \mathrm{m}$ & $9.95 \pm 0.52$ & 12644096 & \\
\hline 2004 Mar. 15 & Spitzer/MIPS & $70 \mu \mathrm{m}$ & $25.7 \pm 1.7$ & 4320256 & Muzerolle et al. (2004) \\
\hline 2004 Oct. 14 & Spitzer/MIPS & $70 \mu \mathrm{m}$ & $30.7 \pm 1.9$ & 12260352 & \\
\hline 2004 Oct. 15 & Spitzer/MIPS ( $(\star)$ & $70 \mu \mathrm{m}$ & $36.6 \pm 3.7$ & 12260608 & \\
\hline 2005 Mar. 1 & Spitzer/MIPS ( $(\star)$ & $70 \mu \mathrm{m}$ & $30.6 \pm 3.1$ & 11570432 & \\
\hline 2005 Mar. 4 & Spitzer/MIPS ( $(\star)$ & $70 \mu \mathrm{m}$ & $32.6 \pm 3.3$ & 11576576 & \\
\hline 2006 Nov. 8 & Spitzer/MIPS & $70 \mu \mathrm{m}$ & $2.7 \pm 0.2$ & 17455360 & \\
\hline 2004 Oct. 15 & Spitzer/MIPS & $55-95 \mu \mathrm{m}$ & & 12260608 & \\
\hline 2005 Mar. 1 & Spitzer/MIPS & $55-95 \mu \mathrm{m}$ & & 11570432 & \\
\hline 2005 Mar. 4 & Spitzer/MIPS & $55-95 \mu \mathrm{m}$ & & 11576576 & \\
\hline
\end{tabular}

Notes. Notes give Spitzer AOR numbers and VLTI baseline parameters. Synthetic photometry derived from the Spitzer spectroscopic data are marked with asterisks. The mid-infrared MIDI and Spitzer spectra are presented in Sect. 3.

visibilities. Although we investigated all calibrator data taken on the nights of our MIDI observations, one should probably not consider those where MACAO was used to estimate the errors of the instrumental visibilities. Note that MACAO was not used for the observations of HD 37160 . Nonetheless, the results for HD 37160 agree well with the other instrumental visibilities (within $1 \sigma$ ) obtained the same night. The beam overlap was good at both epochs, even despite the lack of MACAO support. We 
found that the errors of the correlated fluxes are small (a few percent), but those of the total spectra are higher $(10-15 \%)$ and can change slightly with data reduction parameters. For the sake of simplicity, we applied a $15 \%$ error on the spectra, independent of wavelengths. The errors of $15 \%$ on visibility data (see Fig. 2) are consistent with other observations (cf. Chesneau 2007).

Similarly to Paper I, we derived $8.7 \mu \mathrm{m}$ photometry of V1647 Ori from the acquisition images, using the acquisition images of HD37160 for calibration (see Table 1).

In Paper I we presented the first dataset from 2005 March reduced with MIA 1.3. For the sake of consistency, we rereduced the dataset with MIA 1.6. We discuss the differences between the results produced by the different versions of MIA in Appendix A.

\subsection{Optical and near-infrared photometry}

In addition to the already existing photometric data (AP07), new near-infrared (NIR) $J$-, $H$-, and $K_{\mathrm{s}}$-band observations were carried out in 2007 and 2008 (Table 1). These data can be used to characterize the post-outburst quiescent phase object. The data were obtained using CAIN-2 installed on the $1.52 \mathrm{~m}$ Carlos Sanchez Telescope (TCS) at the Teide Observatory (Tenerife, Canary Islands, Spain). We also observed V1647 Ori in the $I_{\mathrm{J}}$ band with the $82 \mathrm{~cm}$ IAC- 80 telescope at the Teide Observatory and in the $I_{\mathrm{C}}$ band with the $1 \mathrm{~m}$ RCC telescope at the Piszkéstetô station of the Konkoly Observatory. The technique of observation, data reduction, and photometric calibration of these data were identical with our previous TCS, IAC-80, and RCC observations of V1647 Ori and are described in detail in AP07.

\subsection{Spitzer archival data}

V1647 Ori was observed with the Infrared Array Camera (IRAC) onboard the Spitzer Space Telescope at 3.6, 4.5, 5.8, and $8.0 \mu \mathrm{m}$ at four different epochs (Table 1) using the subarray readout mode. Offering exposure times as short as $0.02 \mathrm{~s}$, the subarray mode enables the observation of bright sources that would saturate the detector in other readout modes. IRAC data can be downloaded from the Spitzer Heritage Archive. We considered all IRAC sub_array images that provided more reliable results than the mapping data. These were obtained on 2004 March 7 and the results were published by Muzerolle et al. (2005). The authors derived somewhat $(\approx 10 \%)$ lower fluxes at each observing wavelength. All these observations except for those carried out in 2004 October consist of nine different dither positions with 64 images at each dither position (with size of $32 \times 32$ pixel). They were obtained with an exposure time of $0.1 \mathrm{~s}$ per frame. Four dither positions were used in 2004 October. The data processing started with the Spitzer Science Center (SSC) basic calibrated data (BCD) produced by the pipeline version S18.7. The BCD image cubes of the 64 frames were combined into two-dimensional images using the irac-subcubecollapse IDL routine provided by the SSC. Aperture photometry was performed on the final images at each wavelength using a modified version of the IDLPHOT routines. The aperture radius was set to 3 pixels (pixel scale $11^{\prime \prime} 2$ ), the sky background was computed in an annulus with an inner radius of 3 pixels and a width of 4 pixels. In the course of the sky estimates we used an iterative sigma-clipping method, where the clipping threshold was set to $3 \sigma$. Following the outline of Hora et al. (2008), we also applied an array-dependent photometric correction and a pixelphase correction to the measured flux densities. An aperture correction was then performed using the values published in the
IRAC instrument handbook ${ }^{4}$ (IIH). The flux density values measured at the different dither positions (at each band) were averaged to obtain the final photometry. The final uncertainties were computed by quadratically adding the measurement errors (obtained from the individual flux density values in each band) and an absolute calibration error of $3 \%$ (IIH). No color correction was applied since it would have only a marginal effect $(<1 \%)$ according to our calculations.

V1647 Ori was observed with the Infrared Spectrograph (IRS) of Spitzer at three different epochs. On 2004 October 21 and on 2005 March 24 the target was measured with the shortlow (5.2-14.5 $\mu \mathrm{m}, R \sim 60-127)$, short-high (9.9-19.5 $\mu \mathrm{m}, R \sim$ 600) and long-high $(18.7-37.2 \mu \mathrm{m}, R \sim 600)$ channels, while on 2005 March 11 the short-low and the long-low $(14.0-38.0 \mu \mathrm{m}$, $R \sim 57-128$ ) channels were used. All these data were published by Quanz et al. (2007), and we considered these spectra for our modeling.

V1647 Ori was also observed with the Multiband Imaging Photometer for Spitzer (MIPS, Rieke et al. 2004) in scanmap mode on 2004 March 15 and in photometry-mode on 2004 October 15 and 2006 November 8. The data analysis of the MIPS observations started with the pipeline (S16.1) generated basic calibrated data (BCD) images. At $24 \mu \mathrm{m}$ we performed a flat-field correction and a background-matching using the MOPEX tool (Makovoz \& Marleau 2005). Following the general steps for processing MIPS $70 \mu \mathrm{m}$ data described by Gordon et al. (2007), we performed column-spatial-filtering and time-median-filtering on BCD images obtained at this wavelength. The improved BCD images were subsequently mosaiced using MOPEX. The output mosaic was resampled to 2'.45 and $4^{\prime \prime}$ pixel $^{-1}$ at 24 and $70 \mu \mathrm{m}$. The MIPS $70 \mu \mathrm{m}$ array is affected by nonlinearity at high count rates. To correct for the $70 \mu \mathrm{m}$ nonlinearity effects, we applied the formula described by Dale et al. (2009, see Eq. (1)). At $24 \mu \mathrm{m}$ V1647 Ori is saturated, therefore we used a model PSF to fit the pixels that were still in the linear regime. At $70 \mu \mathrm{m}$ we used a modified version of the IDLPHOT routines to obtain aperture photometry for V1647 Ori. The aperture was placed at the SIMBAD position of the source and the background was estimated in a sky annulus between $39^{\prime \prime}$ and $65^{\prime \prime}$. For images taken at the first two epochs the aperture radius was set to $18^{\prime \prime}$. In the last image, however, the source appeared at the edge of the image, which allowed us to use only a smaller aperture (radius of $12^{\prime \prime}$ ). The aperturecorrection factors, appropriate for a $60 \mathrm{~K}$ blackbody, were derived following the outline of Gordon et al. (2007). The final uncertainties were computed by quadratically adding the internal error and the absolute calibration uncertainty (we adopted $7 \%$, see MIPS data Handbook ${ }^{5}$ ). The final photometry and its uncertainty is listed in Table 1 . The MIPS data in Table 1 are color-corrected (by a factor of 0.91). Our results for $2004 \mathrm{March}$ are higher by $16 \%$ and $46 \%$ at 24 and $70 \mu \mathrm{m}$, compared to those of Muzerolle et al. (2005) because of the different pipeline versions and the additional data reduction steps.

V1647 Ori was also observed using the MIPS SED mode. Low-resolution far-infrared (55-95 $\mu \mathrm{m} ; \lambda / \Delta \lambda \sim 15-25)$ spectra were obtained on 2004 October 15 and on 2005 March 1 and 4. The data reduction of SED observations also started with the BCD images (pipeline version S16.1) and MOPEX was used for the processing steps (combination of data, background removal,

\footnotetext{
4 Version 1.0, http://ssc.spitzer.caltech.edu/irac/ iracinstrumenthandbook/

5 Version 2.0, http://ssc.spitzer.caltech.edu/mips/ mipsinstrumenthandbook/
} 
application of the dispersion solution) and to compile the final images with a pixel scale of $9.8^{\prime \prime}$. An aperture covering 5 pixels was used to extract the spectra from the final images. The aperture correction factors were taken from Lu et al. (2008).

Finally, synthetic $8.0,24$, and $70 \mu \mathrm{m}$ photometry was derived from the IRS and MIPS SED data by convolving the spectra with the corresponding IRAC or MIPS filter profiles. The results are presented in Table 1 .

\section{Results}

\subsection{Optical and infrared light curves}

Figure 1 shows the light curves of V1647 Ori at five optical and infrared wavelengths $(0.8,2.2,8.0,24$, and $70 \mu \mathrm{m})$ between 2004 and 2007. The overall shape of the $I_{C}$ light curve, which is the most complete, can be divided into a plateau (2004 February-2005 September), a rapid fading (2005 October-2006 February), and the subsequent quiescent phase. In addition to the general slow fading during the plateau phase, short timescale low-amplitude variations are also observed. The rapid fading started around the epoch of the second MIDI observations. The available data in the $K_{\mathrm{s}}$ band may suggest a light curve similar to that of $I_{\mathrm{C}}$, but with a smaller amplitude. Although the light curves at 8,24 , and $70 \mu \mathrm{m}$ are even more sparsely sampled, they are not inconsistent with the shape of the $I_{\mathrm{C}}$ band light curve. We note that at $70 \mu \mathrm{m}$, the object became $\sim 20 \%$ brighter between the 2004 March peak and 2004 October. During the same period, the IRAC fluxes decreased by a factor of 2. This suggests a time shift between the peak brightnesses at the different wavelengths. The cause of such a shift at $70 \mu \mathrm{m}$ is unclear.

Comparing the flux values obtained in 2006 to the preoutburst ones (interpolated in wavelength in Fig. 1 of Ábrahám et al. 2004), we conclude that V1647 Ori returned to quiescence after 2005. The 2007 fluxes at 8 and $24 \mu \mathrm{m}$ are even lower than the respective pre-outburst values. This could either be due to variability in the quiescent phase (similar as seen in the preoutburst $I_{C}$ data of Briceño et al. 2004), might be related to the beam differences of the different instruments, differences in the photometric systems, or might even indicate a different object structure after the outburst.

\subsection{Mid-infrared interferometry}

Figure 2 shows the calibrated visibilities obtained in 2005 March and September. The baselines of the two observations are very similar (Table 1), thus the two visibility curves can be directly compared. The difference indicates a significant change in the geometry of the mid-infrared emitting region of V1647 Ori in the six months that elapsed between the two observations. As a first approximation, we considered Gaussian brightness distributions to estimate the characteristic size of the mid-infrared emitting region defined as the FWHM of the Gaussian. Although the object is much more resolved at the second epoch, and accordingly the Gaussian assumption is less adequate for size estimation, we converted the visibilities to the FWHM, which provided a simple comparison of the interferometric data. Single Gaussians with a $F W H M=10$ and 16 mas, equivalent to 4.0 and 6.4 AU at the distance of $400 \mathrm{pc}$, respectively, fit both visibility datasets well longward of $\sim 10 \mu \mathrm{m}$. By fitting Gaussians to the visibilities, characteristic sizes of 5-7 mas (2.0-2.8 AU) and 11-13 mas (4.4-5.2 AU) can be estimated at shorter wavelengths for the two epochs. The object was less resolved at wavelenghts shortward of $\sim 10 \mu \mathrm{m}$ at both epochs, probably due

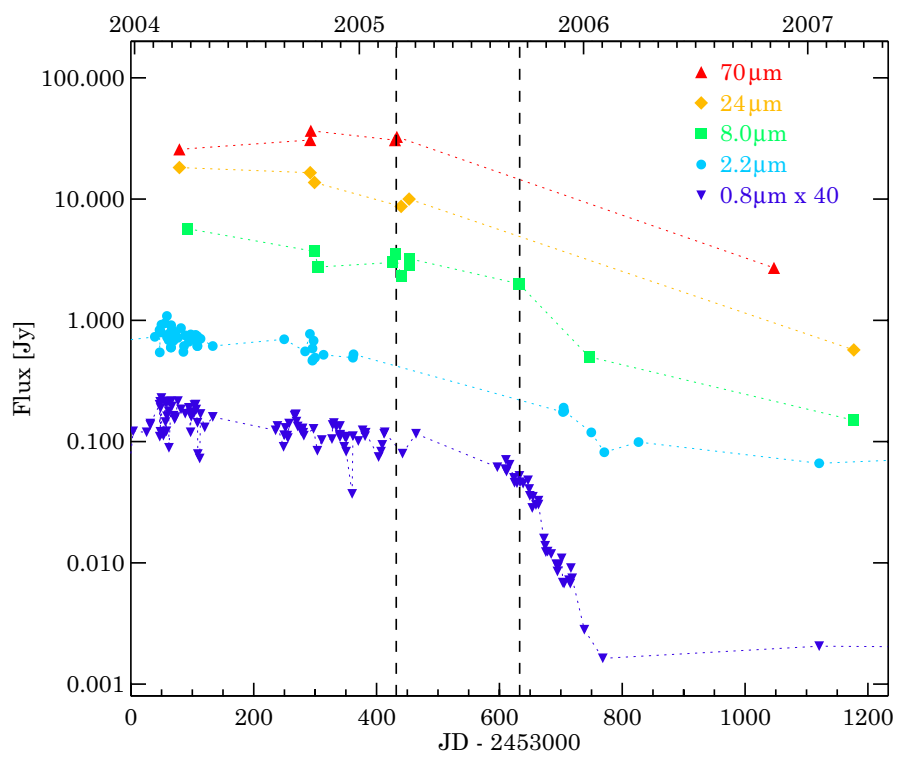

Fig. 1. Light curves of V1647 Ori. The vertical dashed lines denote the epochs of the MIDI observations. $I_{\mathrm{C}}(0.8 \mu \mathrm{m})$ and $K_{\mathrm{s}}(2.2 \mu \mathrm{m})$ band data are from AP07 and this paper. The $I_{\mathrm{C}}$ data are scaled up by a factor of 40 for the sake of better plotting. $8.0 \mu \mathrm{m}$ data are from Muzerolle et al. (2005), Paper I, Quanz et al. (2007), this paper (obtained from MIDI acqusition data), and Aspin et al. (2008, extrapolated from their $N^{\prime}$ band data). $24 \mu \mathrm{m}$ data are from this paper and Aspin et al. (2008) (extrapolated from their $Q_{a}$ band data). $70 \mu \mathrm{m}$ data are from this paper.

to an inner warm part of the system. Because the shape of the visibility curves is similar to those observed for other young stellar objects (YSOs) with MIDI (e.g., Leinert et al. 2004; Quanz et al. 2006; Ratzka et al. 2007), we can assume that they have similar circumstellar structures, which means that the radiation from the inner edge of the disk can dominate at short wavelengths.

Similarly to Paper I, we do not see any sinusoidal variations in the spectrally resolved visibilities that are potentially caused by a companion. Since the baselines of the two observing runs are very similar, we can only repeat our earlier finding that the shape of the new visibility curve suggests that no companion whose separation is between about 10 and a few hundred AU and has a brightness ratio higher than $10 \%$ is present at the measured position angle .

Our two $8-13 \mu \mathrm{m}$ MIDI spectra are shown in Fig. 2. Although the absolute level of the mid-infrared spectra decreased by a factor of 2 between the two epochs, the shapes of the two spectra are similar. In Fig. $2 \mathrm{~d}$ we plot the first MIDI spectrum together with the two IRS spectra obtained in 2005 March. Variations on weekly timescales are clearly present. Quanz et al. (2007) found weak silicate emission in the IRS spectra. Although the ozone band can affect the spectra at $10 \mu \mathrm{m}$, this weak feature might also be present in the MIDI spectra.

The uncorrelated spectra are calculated as the difference of the total and the correlated ones. The correlated and uncorrelated spectra of an object are dominated by radiation from different circumstellar regions: the inner regions of a few AU size and the outer parts (van Boekel et al. 2004). In the case of V1647 Ori, most of the mid-infrared emission originates from the inner compact zone of the circumstellar environment, the correlated spectrum being higher than the uncorrelated spectrum. The correlated fluxes decreased significantly, by approximately $60 \%$ between the two MIDI observations, while the relatively low uncorrelated fluxes increased by about $30 \%$ longward of $10 \mu \mathrm{m}$, 

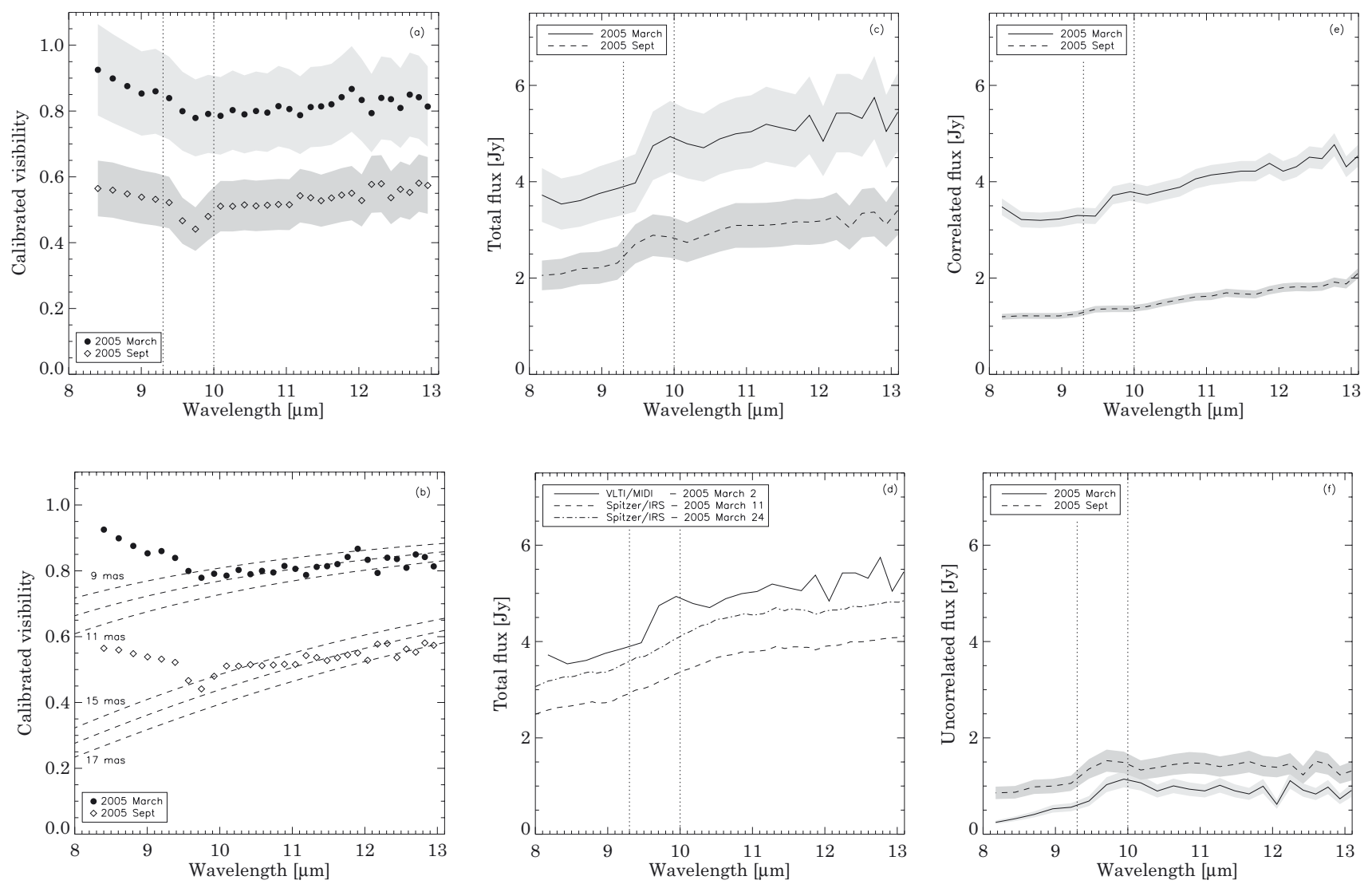

Fig. 2. Calibrated visibilities a) and Gaussian size estimates b). MIDI spectra are shown in panel c). The MIDI and Spitzer/IRS spectra from 2005 March are compared in panel d). The errors of the Spitzer spectra ( 10\%) are not shown for the sake of clarity. Correlated spectra (i.e., inner disk spectra, panel e)) and uncorrelated spectra (i.e., outer disk spectra, panel f)) of V1647 Ori are also shown at two epochs. The baselines of the two observations are very similar, so the MIDI data of the two epochs can be compared. The shaded areas mark the errors in the panels a), c), e), f)). The vertical dotted lines show the ozone band that could affect the ground-based observations.

and by up to $70 \%$ shortward of $10 \mu \mathrm{m}$ (Fig. 2f). These findings may suggest either a structural change in the circumstellar environment of the object or that the fading of the inner and the outer parts of the mid-infrared emitting regions of V1647 Ori occured on different timescales. The correlated spectra look featureless; the weak silicate emission seems to be associated with the outer parts of the system.

\subsection{Spectral energy distribution}

We constructed SEDs of V1647 Ori for several different epochs to study its circumstellar environment. These epochs were 2004 March (peak of the outburst), 2004 October (plateau phase 1), 2005 March (plateau phase 2), 2005 September (shortly before the rapid fading). The latter two epochs are those of the MIDI observations. Due to the lack of simultaneous optical and near-infrared data, we included data points in the SEDs obtained within a period of a few weeks of the nominal date. We also compiled a combined SED from pre- and post-outburst quiescence data (cf. Aspin et al. 2008). Because of the sparse time coverage of observations, i.e., no simultaneous SED, we merged all available measurments and plotted all pre- and postoutburst data together in the quiescent SED. The five SEDs are plotted in Fig. 3. The Spitzer IRS and MIPS SED spectra are also plotted. The absolute errors of these spectra are $\sim 10 \%$. For reasons related to the radiative transfer modeling, which is described in Sect. 4, we consider data only shortward of $100 \mu \mathrm{m}$.
Submillimeter and millimeter data are used only for the estimation of the mass of the system (see Sect. 4.1.1).

\section{Modeling}

In the following we fit both the SEDs and the visibilities using a radiative transfer code to provide a model of the circumstellar environment and the heating mechanisms. Our strategy for fitting the data was as follows. First we set up a reference model that fits the 2005 March data (Sect. 4.2). We chose this epoch to serve as a starting point because our observational data set is most complete here. Then we modified this initial setup as necessary to fit the data of the other epochs (Sect. 4.3). The significant change of the MIDI visibilities might be explained by different scenarios, which are discussed separately in Sect. 4.4.

We note that first we tried to adopt the simplistic disk model of the V1647 Ori system presented in our Paper I for all epochs. Although the SED models were satisfactory, the model cannot reproduce the low visibility values measured in September 2005.

\subsection{General overview of the model}

For the radiative transfer modeling we used the Monte Carlo code MC3D (Wolf et al. 1999; Wolf 2003; Schegerer et al. 2008). We assumed an axial symmetry of the circumstellar environment, therefore we used a two-dimensional geometry in polar coordinates $(r, \theta)$.

The model system consists of a central star, an inner accretion (gas) disk, and a dusty outer disk embedded in an envelope, 


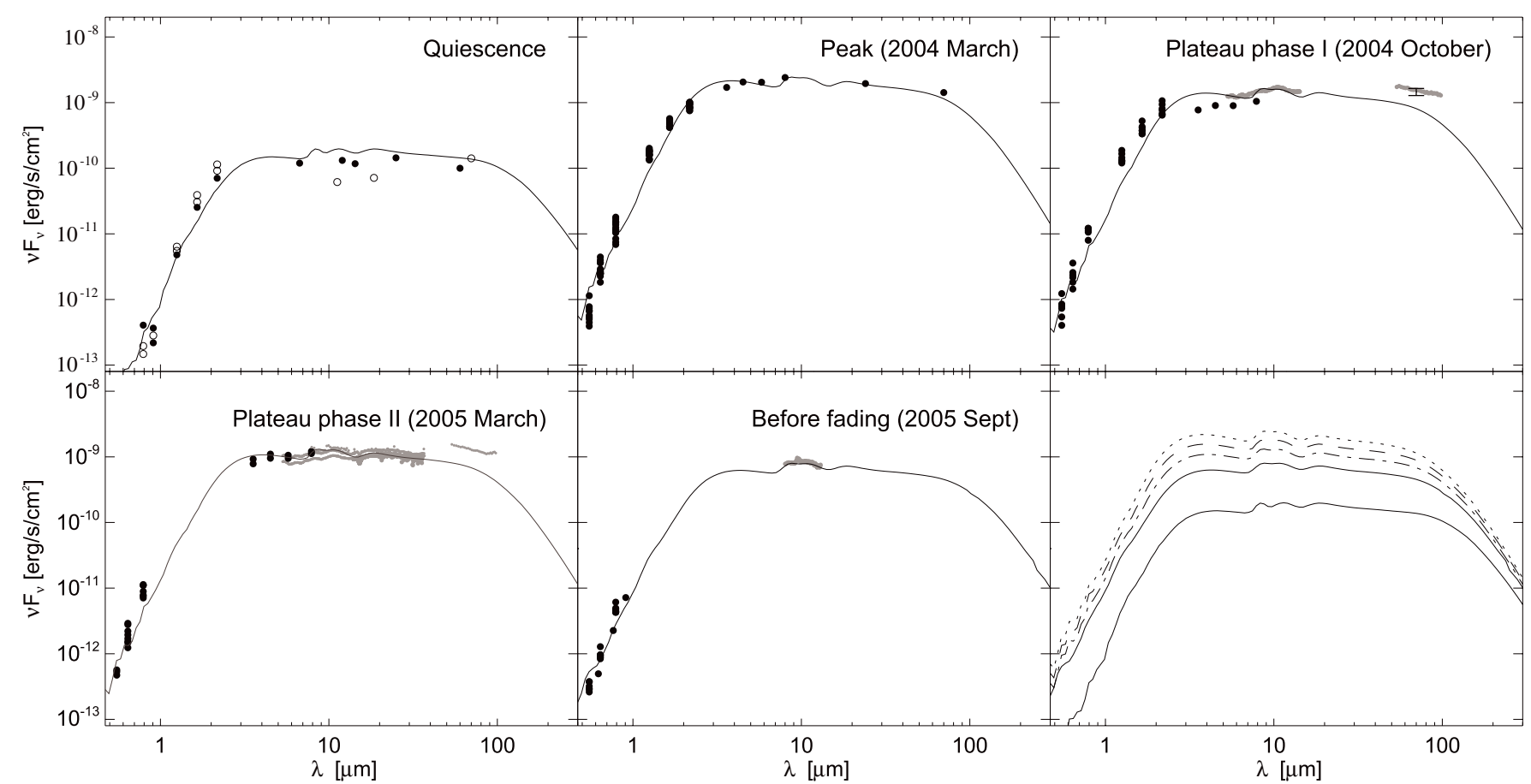

Fig. 3. Spectral energy distributions of V1647 Ori at five different epochs. Because of the short timescale variability at the optical and near-infared wavelengths, we present photometry from between 2004-02-01 and 2004-04-30 (2004 March), 2004-10-01 and 2004-10-31 (2004 October), 2005-02-01 and 2005-04-30 (2005 March) and 2005-09-03 and 2005-10-03 (2005 September) in these figures. Our models to the SEDs, where the inner radius of the dust disk is set to $0.5 \mathrm{AU}$ in quiescence and $0.7 \mathrm{AU}$ in all outburst epochs, are overplotted with solid lines (see Sect. 4). Between the outburst epochs only the accretion rate was changed here. Note that the model for 2005 September shown here does not fit the MIDI data (see Fig. 5 and Sect. 4.4). The last panel shows all models. The decrease of the brightness of the object with time, from peak (top) to quiescence (bottom) is continuous. References: quiescence - Ábrahám et al. (2004), Paper I, Aspin et al. (2008); peak (2004 March) - AP07, McGehee et al. (2004), Muzerolle et al. (2005); plateau phase I (2004 October) - AP07, this work, Quanz et al. (2007); plateau phase II (2005 March) AP07, this work, Quanz et al. (2007); before the rapid fading (2005 September) - Aspin \& Reipurth (2009), this work.

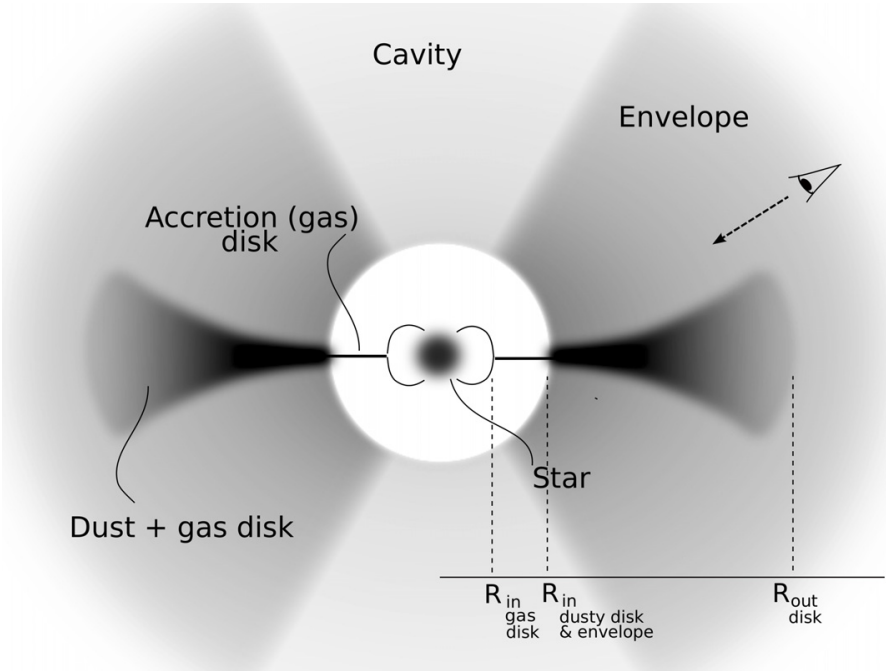

Fig. 4. Schematic picture of the model geometry of the object (not to scale). The line of sight crosses through the envelope. The inclination of the disk is $\vartheta \approx 60^{\circ}$, the opening angle of the conical cavity is $50^{\circ}$ (AP07).

from which a cone-shaped cavity is cut (Fig. 4). For the structure of the disk we assumed the density profile to be like the one used by Wood et al. (2002),

$\rho_{\text {disk }}=\rho_{0, \text { disk }}\left(\frac{R_{*}}{\varpi}\right)^{\alpha_{\text {disk }}} \exp \left\{-\frac{1}{2}\left[\frac{z}{h(\varpi)}\right]^{2}\right\}$, where $\varpi$ is the radial coordinate in the disk midplane, $z$ is the vertical height and $\rho_{0}$ is determined from the mass of the disk. The scale height $h(\varpi)$ increases with radius as,

$h=h_{0}\left(\frac{\varpi}{100 \mathrm{AU}}\right)^{\beta}$,

where $h_{0}$ is the scale height at a radial distance of $100 \mathrm{AU}$. The density profile of the envelope is spherically symmetric:

$\rho_{\mathrm{env}}=\rho_{0, \mathrm{env}}\left(\frac{R_{*}}{r}\right)^{\alpha_{\mathrm{env}}}$.

Here $r$ gives the distance from the star, and where $\rho_{0 \text {,env }}$ is defined as $f \rho_{0 \text {, disk }}$ at a certain arbitrary point of the disk and the envelope. The value of the constant $f$ has no physical meaning, it is set so as to separately control the mass of the disk and the envelope. The transition between these two components is assumed to be smooth. In vertical direction the envelope starts where the density of the disk decreases to the density of the envelope. In the cavity we used a density distribution identical to that of the envelope, but less dense by a factor of $10^{-6}$.

The temperature distribution of the system is determined by the heating sources: the central star, the heated dust grains, emitting blackbody radiation, and the accretion, consisting of an $\alpha$-type disk concentrated on the midplane and a hot spot on the stellar suface (for details of accretion effects see Schegerer et al. 2008). The accretion process is characterized by three parameters, the accretion rate $\dot{M}$, the temperature $T_{\text {spot }}$ of the accreting region on the surface of the star, and the magnetic truncation radius $R_{\text {trunc }}$. After calculating the temperature distribution, 
the SED and a projected image of the system is produced at an inclination angle $\vartheta$ with a ray-tracer.

The circumstellar disk in our model is embedded in a significantly larger envelope that stretches out far beyond the disk, to $3000 \mathrm{AU}$, in accordance with the mm-images from Tsukagoshi et al. (2005). The inclination of the disk was fixed to be $\vartheta \approx 60^{\circ}$ and we set the opening angle of the cavity to $50^{\circ}$ (AP07), thus the line of sight to the star crosses the envelope. Although the midinfrared ice features indicate the presence of foreground material (Vacca et al. 2004; Rettig et al. 2005; Quanz et al. 2007), the ice can also be in the outer regions of the extended envelope around V1647 Ori. Therefore we fixed the interstellar extinction to be $A_{\mathrm{V}}=0$ and assumed that all extinction is circumstellar.

The $10 \mu \mathrm{m}$ silicate feature is too weak to determine the dust composition via model-fitting. Therefore we considered standard interstellar dust, a mixture of $62.5 \%$ astronomical silicate, and $37.5 \%$ graphite (the optical parameters and grain size distribution were taken from Weingartner \& Draine 2001). The shape of the continuum-subtracted features (Quanz et al. 2007) and the presence of an envelope support the plausibility of this assumption. We used spherical dust grains, with a power-law size distribution $n(a) \propto a^{-3.5}$, for $a_{\min } \leq a \leq a_{\max }$, where $n(a)$ is the number of dust particles with a radius $a$. For the minimum and maximum grain size we used $0.005 \mu \mathrm{m}$ and $0.25 \mu \mathrm{m}$. To limit the number of free parameters in the model fitting, we fixed $a_{\min }$ and $a_{\max }$. Note that increasing the maximum grain size to a few $\mu \mathrm{m}$ would decrease the silicate emission, but would not change the model SEDs significantly. The gas-to-dust mass ratio is assumed to be 100 , while the grain mass density is set to $\rho_{\mathrm{g}}=2.7 \mathrm{~g} \mathrm{~cm}^{-3}$. We have found that with the selected grain size distribution we underestimate the submm-to-mm flux in all our models, unless we assume a disk mass of $\approx 1 M_{\odot}$, which would result in gravitational instabilities. In addition, the submm-mm slope of the model is steeper than that of the measured data, which leads to the conclusion that the system possibly contains larger grains than the maximum size used. However, we think the larger grains are within the inner regions of the disk closer to the midplane due to sedimentation, affecting the SED significantly only in the (sub-)mm regime, since this region is optically thick at shorter wavelengths. Consequently, we consider our model to be valid shortward of $\sim 100 \mu \mathrm{m}$.

We estimated the mass of the system from the $1.3 \mathrm{~mm}$ flux (Lis et al. 1999), using the method described in Beckwith et al. (1999). Assuming $T=50 \mathrm{~K}$ and $\kappa_{v}=0.02 \mathrm{~cm}^{2} \mathrm{~g}^{-1}$, we found that the total mass of the circumstellar matter is $M_{\mathrm{tot}}=0.045 M_{\odot}$. The estimate is similar to that of Andrews et al. (2004). We considered this estimate as a fixed parameter during our model-fitting.

\subsection{The reference model: 2005 March}

As a first step we fitted the available photometric and interferometric data for the 2005 March epoch. Since the SED is not composed of results of simultaneous observations and the object is variable on short timescales (see Sect. 3.3), we consider ranges of data at certain wavelengths ${ }^{6}$. Such a dataset prevents us from calculating correct meaningful $\chi^{2}$ values and comparing different models in a mathematical way. Instead, we evaluated the plausibility of the models by visual inspection of the fits - as done in most of the cases, see e.g., Schegerer et al. (2008), Ratzka et al. (2009), van Boekel et al. (2010), Juhász et al. (2012). The model-fitting in the latter two cases

\footnotetext{
6 This holds also for the other epochs.
}

is very similar to our case because the objects studied by the authors (T Tau and EX Lup, respectively) are also variable and the authors fitted merged SED datasets, as we did for the different epochs.

We set the derived model parameters against results found in the literature to filter out unphysical models that still showed good fits. The model has many parameters, and a good fraction of these cannot be determined or constrained by measurements. We attempted to fit the data with the extreme cases of envelopeonly and disk-only models, but we conclude that neither of the two components can be neglected. The parameters of the circumstellar environment are not clearly defined by the models because the parameters of the envelope and the disk are not independent (e.g., Natta 1993). To reduce the degeneracy we fixed additional system parameters and tried to consider canonical values. We set the outer radius of the disk to $500 \mathrm{AU}$ and the value of $\alpha_{\text {env }}$ to -1.5 (Shu 1977). To reduce the number of free parameters even more, we set the inner radii of the disk and the envelope to be identical. The investigated parameter ranges are listed in Table 2. In the following we present our results with the fixed parameters, although our conclusions and the trends seen - based on the models we propose - are also valid for slightly different setups of the envelope-disk system. Therefore we do not present best-fit models here, but a series of plausible good models.

During the fitting procedure different $\dot{M}$ and $R_{\text {in }}$ values were considered, with step sizes of $10^{-7} M_{\odot} / \mathrm{yr}$ and $0.05 \mathrm{AU}$, respectively. The step sizes were 5-10\% maximum for all other epochs. For the reference epoch we found that the accretion rate is $3.5 \times 10^{-6} M_{\odot} / \mathrm{yr}$ and the inner radii of the disk and the envelope are $R_{\text {in }}=0.7 \mathrm{AU}$. Since the line-of-sight to the star crosses the envelope but not the disk (Fig. 4), the parameters of the envelope (mass, $R_{\text {in }}$ and $\alpha_{\text {env }}$ ) strongly determine the fits in the optical-NIR regime. The thick envelope increases the extinction in the system in order to decrease the otherwise overestimated optical-NIR model fluxes to the measured values ${ }^{7}$, and it also helps reducing the height of the intrinsically strong $10 \mu \mathrm{m}$ silicate emission feature. The parameters of our model are given in Table 2, the model can be seen in the upper panels of Fig. 5. The visibilities are somewhat underestimated. A better fit could be achieved, but at the cost of a poorer SED-fit.

Comparing the disk and envelope parameters of our model with the results of similar modeling efforts of embedded YSOs in the literature (e.g., Whitney et al. 2003), we may conclude that the circumstellar environment of V1647 Ori is rather typical, and no structural features directly linked to the eruptive nature of the object can be identified. With our model we could describe the system with a parameter setup that is plausible for young stellar objects.

\subsection{Modeling the variations during outburst}

In order to fit the SEDs of the other epochs, we used the 2005 March model as an initial setup. At first sight, the SEDs differ only in their absolute levels, but their shapes are very similar (Fig. 3). Therefore, we attempt to adjust the shift between the levels by the variation of the accretion rate. This way we test if it is possible to keep the geometry of the circumstellar environment identical, and only change the illumination of the system as the outburst proceeds. Additionally, we tried to vary only the smallest number of parameters possible between the different epochs and restricted ourselves to applying modifications

7 Note the absence of NIR photometric data in 2005 (Figs. 1 and 3). 
Table 2. Parameters of the best-fitting model for 2005 March.

\begin{tabular}{|c|c|c|c|}
\hline Parameters & Final model & References for fixed parameters & Ranges for fit \\
\hline \multicolumn{4}{|l|}{ Stellar parameters } \\
\hline Temperature $\left(T_{\text {star }}\right)$ & $3800 \mathrm{~K}$ & Aspin et al. (2008) & \\
\hline $\operatorname{Mass}\left(M_{\text {star }}\right)$ & $0.8 M_{\odot}$ & Aspin et al. (2008) & \\
\hline $\operatorname{Radius}\left(R_{\text {star }}\right)$ & $3.25 R_{\odot}$ & Aspin et al. (2008), recalculated & \\
\hline Interstellar visual extinction $\left(A_{\mathrm{V}}\right)$ & $0 \mathrm{mag}$ & & \\
\hline \multicolumn{4}{|l|}{ Circumstellar disk parameters } \\
\hline Inner radius of dusty disk $\left(R_{\mathrm{in}, \mathrm{disk}}\right)$ & $0.7 \mathrm{AU}$ & & $0.2-1.5 \mathrm{AU}$ \\
\hline Outer radius of dusty disk $\left(R_{\text {out,disk }}\right)$ & $500 \mathrm{AU}$ & & \\
\hline Scale height at $100 \mathrm{AU}\left(\mathrm{H}_{0}\right)$ & $15 \mathrm{AU}$ & & $3-25 \mathrm{AU}$ \\
\hline Flaring index $(\beta)$ & 1.2 & & $1-9 / 7$ \\
\hline Exponent of radial density profile $\left(\alpha_{\text {disk }}\right)$ & -1.75 & & $-2.25--1.5$ \\
\hline Total mass of disk and envelope $(M)$ & $0.045 M_{\odot}$ & estimated & \\
\hline Distance $(d)$ & $400 \mathrm{pc}$ & Anthony-Twarog (1982), AP07 & \\
\hline Inclination $(\vartheta)$ & $60^{\circ}$ & AP07 & \\
\hline \multicolumn{4}{|l|}{ Circumstellar envelope parameters } \\
\hline Inner radius of dusty envelope $\left(R_{\mathrm{in}, \mathrm{env}}\right)$ & $0.7 \mathrm{AU}$ & & $0.05-1.0 \mathrm{AU}$ \\
\hline Outer radius of dusty envelope ( $\left.R_{\text {out,env }}\right)$ & $3000 \mathrm{AU}$ & Tsukagoshi et al. (2005) & \\
\hline Exponent of radial density profile $\left(\alpha_{\text {env }}\right)$ & -1.5 & & \\
\hline \multicolumn{4}{|l|}{ Parameters for the accretion } \\
\hline Accretion rate $(\dot{M})$ & $3.5 \times 10^{-6} M_{\odot} / \mathrm{yr}$ & & $2-10 \times 10^{-6} M_{\odot} / \mathrm{yr}$ \\
\hline Magnetic truncation radius $\left(R_{\text {trunc }}\right)$ & $5 R_{\mathrm{star}}$ & Calvet \& Gullbring (1998) & \\
\hline Temperature of the hot spot $\left(T_{\text {spot }}\right)$ & $6500 \mathrm{~K}$ & Calvet \& Gullbring (1998) & \\
\hline
\end{tabular}

Notes. The fitted parameters are shown in italics.

that could be the result of a realistic process and may occur in such a short period of time. Significant changes, affecting a large part of the disk or a complete restructuring, could happen only on longer timescales (Chiang \& Goldreich 1997).

According to the standard picture of eruptive YSOs (HK96), the accretion is the main energy source in the system in outburst, while its contribution in quiescence is less pronounced. The accretion rate is expected to increase from the pre-outburst phase to the peak of the outburst and decrease during the eruption as the source is fading. The modeling results confirmed this expectation. We found that the accretion was strongest at the peak brightness of 2004 March $\left(7 \times 10^{-6} M_{\odot} / y r\right.$, see Table 3$)$, and gradually weakened as the source approached the end of its flareup, but still accreted strongly in quiescence $\left(3 \times 10^{-7} M_{\odot} / \mathrm{yr}\right)$. The fit to the mid-infrared part of the SED depends mainly on the accretion rate. The change found in the accretion rate is solid, since the fading can be seen clearly at these wavelengths (see Fig. 3). The strategy of changing the accretion rate worked for the whole SEDs of the outburst epochs, but failed to reproduce the second MIDI interferometric data from 2005 September (see Sect. 4.4 and the middle lower panel of Fig. 5). Lowering the accretion rate to $\approx 5 \%$ of the peak value reduced the fluxes considerably at all optical-IR wavelengths in quiescence. This model fits the mid-infrared part of the SED well, but the excess of the model in the optical-NIR part of the SED indicates that the accretion rate is not the only parameter that must be adjusted.

The inner radii of the dusty components in the reference model are set to $0.7 \mathrm{AU}$, which provides good fit for the other two outburst epochs in 2004. Accordingly, the geometry of the inner part of the system was unchanged during the plateau phase. For quiescence, we found the best fit by using $R_{\text {in,qui }}=0.5 \mathrm{AU}$, which is the radius of both the dust disk and envelope. The variation of the inner radii of the disk and the envelope indicates that a dynamical process worked immediately after the outburst, which enlarged the inner dust-free hole from 0.5 to $0.7 \mathrm{AU}$ on a timescale of a few months. The derived $R_{\text {in }}=0.5$ and $0.7 \mathrm{AU}$ radii correspond to $1000 \mathrm{~K}$ and $1500 \mathrm{~K}$ in quiescent state and at the peak brightness phase, respectively. These temperature values are close to the canonical dust sublimation temperatures ( $1300-1600 \mathrm{~K})$. However, moving the inner radii of the quiescent model inward to the corresponding sublimation radii, the model underestimates the optical data points due to the increased extinction. Although similar deviations of the radii were found at other eruptive young systems (e.g., Sipos et al. 2009; Juhász et al. 2012; Eisner et al. 2011), the cause of this larger inner radius is not clear. Varying parameters of the models are shown in Table 3.

\subsection{September: the puzzling MIDI data}

For the 2005 March and September epochs the MIDI visibilities give additional constraints for the system parameters. We attempted to fit the 2005 September SED and visibility values using the same method followed above. We kept the inner radii unchanged $\left(R_{\mathrm{in}}=0.7 \mathrm{AU}\right)$ and changed the accretion rate between the two MIDI epochs from $3.5 \times 10^{-6} M_{\odot} / y r$ to $2.0 \times 10^{-6} M_{\odot} / y r$. Due to the fading of the central source the mid-infrared emitting region is smaller and results in slightly increased visibilities. This is just the opposite of what the measurements show (shown with open triangles in Fig. 5, lower middle panel). Clearly, although for the SEDs our first strategy of changing only the accretion rate and the inner radii of the dust disk and the envelope works, it cannot account for the changes in the visibilities.

In the framework of our static radiative transfer models the geometry of the circumstellar environment also had to undergo changes in the inner regions of the disk-envelope system to explain the lowering of the visibility points. The change has to take place in the inner regions of the system, because the dynamical timescales for changing the structure of the environment farther out is much longer than the half-year elapsed between the two MIDI observations.

What that might help to explain the changes in the visibility data is to assume that due to the increased heat - because of 

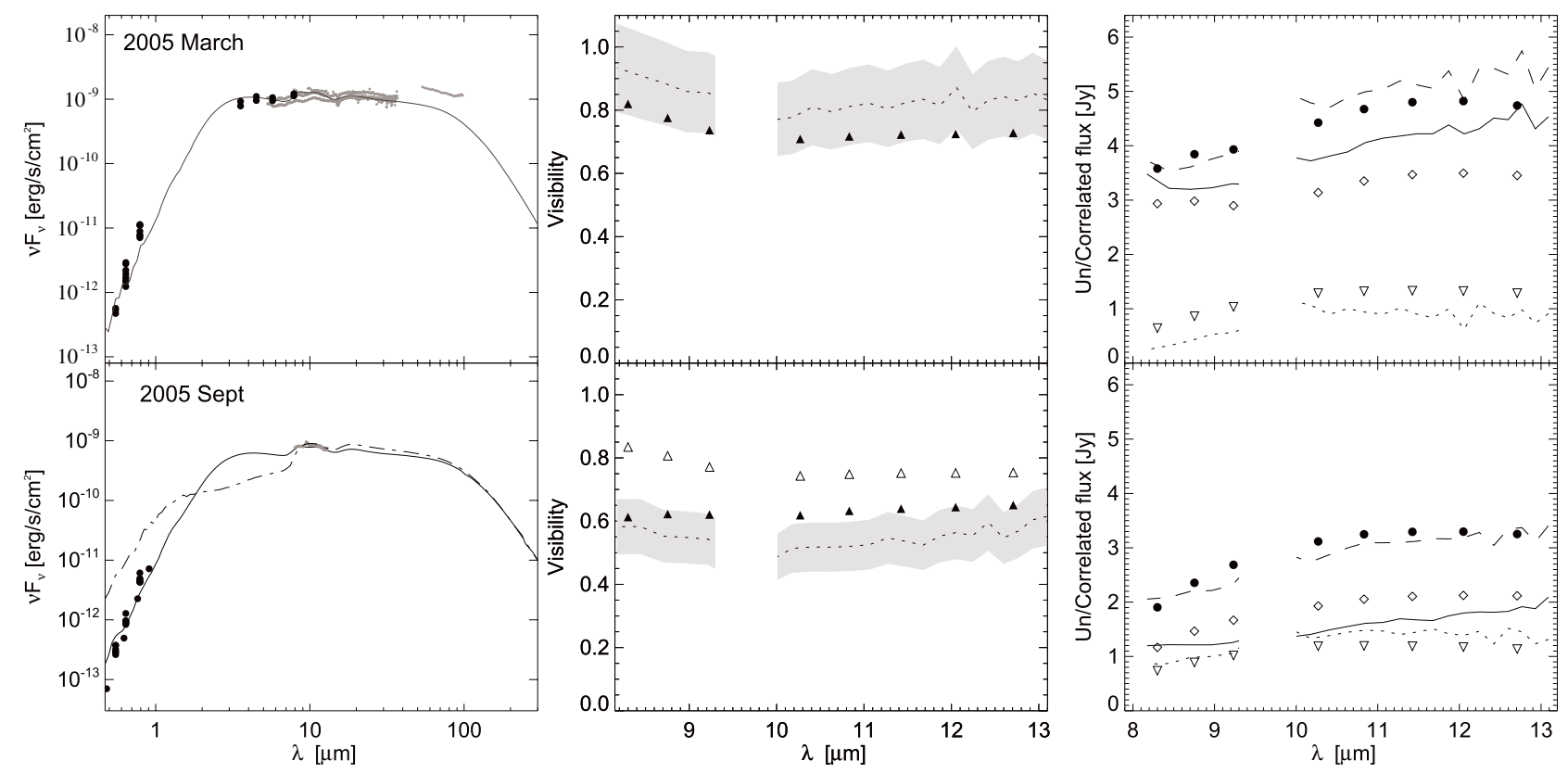

Fig. 5. Our models for the 2005 March and September epochs are shown in the top and bottom row. The SEDs and the calibrated visibilities are shown in the left and middle columns. Model visibilities were calculated at eight different wavelengths. In the bottom left panel the solid line, in the bottom middle panel the open triangles show the model where only the accretion rate was decreased relative to the reference model. This model fits the SED quite well, but not the visibilities. The dash-dotted line (bottom left panel) and the filled triangles (bottom middle panel) show the model where the inner radius of the envelope was also increased. As an alternative interpretation of the mid-infrared fits, the observed mid-infrared total, correlated and uncorrelated MIDI spectra (dashed, solid and dotted lines, respectively) and the corresponding model values (filled circles, diamonds and downward pointing open triangles, respectively) are shown in the right column. The lower right panel refers to the case where the inner radius of the envelope increased well beyond the dust sublimation radius, inducing larger changes in visibilities than the ones described earlier. $R_{\text {in,disk }}$ was kept unchanged. Data probably affected by the atmospheric ozone layer are not shown.

the enhanced accretion - the scale height of the regions close to the inner edge of the disk increases in the first phase of the outburst. By introducing such a region to the system and varying the scale height of the inner part of the disk, i.e., a variable puffed-up inner rim, we also see variations in the visibilities. The visibilities are higher if the scale height increases. A higher inner rim means an additional hot component in the system, which increases the visibilities. However, this rim also casts more shadow on the disk, thus certain regions behind the rim will not be illuminated, which might also affect the visibilities. We tested several geometries of the disk to see how strong the effect is and found that this process could only account for approximately one quarter of the observed decrease, i.e., $0.05-0.08$ in the visibilities. Therefore we do not discuss these models further.

Another straightforward idea is to increase the inner radii of the dusty components from March to September, which we expect to have the effect of increasing the mid-infrared emitting region, i.e., making the source more resolved. The inner radii of the envelope and the disk were changed previously and seem to be the parameters that could vary the easiest (compared to disk scale height or density profile exponents). However, any transportation mechanism that removes material is much more effective on the significantly less dense envelope, and would move its inner radius much farther out. Therefore we do not consider any change in the disk in the following.

In our model that fits the visibilities, the dusty envelope starts only at 3.0 AU, and the matter removed from the inner regions is piled up in a thin shell close to the inner edge of the envelope. This distance corresponds to $\approx 600 \mathrm{~K}$ temperature. By moving the inner edge outwards, the infrared flux increased slightly, so we had to adjust the accretion rate again, i.e., lowered to $1.6 M_{\odot} / \mathrm{yr} \times 10^{-6}$. Moving matter outwards, and creating a shell of matter has the additional effect of reducing the extinction, thus increasing the optical-near infrared flux. As the results show in Fig. 5, improving the fit to the visibilities deteriorated the SED-fit. The extinction in this model dropped to $A_{\mathrm{V}}=11.5 \mathrm{mag}$, which would have led to an unobserved brightening of V1647 Ori (cf. Fig. 2). Although the accretion luminosity decreased in this period, it could hardly compensate for the effect of $\Delta A_{\mathrm{V}}$. The uncertainty of the optical part of our model for 2005 September prevents us from analyzing of the evolution of the extinction at this epoch. We attempt to find the mechanism that can produce the dramatic variation of the envelope radius between March and September 2005 in Sect. 5.4.

\section{Discussion}

\subsection{The varying accretion rate}

The peak accretion rate value we found is on the same order of magnitude as the $5-10 \times 10^{-6} M_{\odot} / \mathrm{yr}$ values derived from $\mathrm{Br} \gamma$ measurements (AP07) and modeling (Muzerolle et al. 2005). During the plateau phase of the outburst, as the object slowly faded, our model accretion rate slightly decreased. Both observations and modeling indicate that $\approx 10^{-5} M_{\odot}$ material was accreted onto the star during the outburst.

The derived accretion rates fit in the canonical picture of eruptive low-mass YSOs. In quiescence the accretion rate of our model $\left(3.0 \times 10^{-7} M_{\odot} / \mathrm{yr}\right)$ is similar to those of class I YSOs $\left(10^{-9}-10^{-7} M_{\odot} / y r\right.$, White \& Hillenbrand 2004) or class II objects $\left(10^{-9}-10^{-6} M_{\odot} / y r\right.$, Gullbring et al. 1998, Hartmann et al. 1998). During the outburst it is $3.0-7.0 \times 10^{-6} M_{\odot} / \mathrm{yr}$, higher than that of quiescent low-mass YSOs, comparable to that of FUors $\left(10^{-6}-10^{-4} M_{\odot} / y r, H K 96\right)$. Intermediate values 
Table 3. Varied model parameters for different epochs.

\begin{tabular}{lccccc}
\hline \hline \multirow{2}{*}{ Parameters } & \multicolumn{2}{c}{2004} & \multicolumn{2}{c}{2005} & $2003 / 06$ \\
& Mar. & Oct. & Mar. & Sept. & quiescent \\
\hline$\dot{M}\left(M_{\odot} / \mathrm{yr} \times 10^{-6}\right)$ & 7.0 & 5.5 & 3.5 & 1.6 & 0.3 \\
$R_{\text {in,disk }}(\mathrm{AU})$ & 0.7 & 0.7 & 0.7 & 0.7 & 0.5 \\
$R_{\text {in,env }}(\mathrm{AU})$ & 0.7 & 0.7 & 0.7 & 3.0 & 0.5 \\
$A_{\mathrm{V}}(\mathrm{mag})$ & 18.9 & 18.9 & 18.9 & 11.5 & 23.4 \\
\hline
\end{tabular}

are expected for EXors (HK96). Juhász et al. (2012) derived $2.2 \times 10^{-7} M_{\odot} / \mathrm{yr}$ for the prototype EXor, EX Lupi, at its outburst peak in 2008 .

In the quiescent model we derived a similar accretion rate value as AP07 and Muzerolle et al. (2005) found, i.e., a few times $10^{-7} M_{\odot} /$ yr. Aspin et al. (2008) claimed, based on the strength of the $\mathrm{Br} \gamma$ emission line, that the accretion rate was somewhat higher $\left(\approx 10^{-6} M_{\odot} / \mathrm{yr}\right)$ after the outburst. However, the estimates of the accretion rate derived from the Br $\gamma$ measurements strongly depend on the applied value of $A_{\mathrm{V}}$. Aspin et al. (2008) considered higher visual extinction than, e.g., AP07.

As a final refinement to fit the NIR color variations, we also had to modify the parameters describing the accretion characteristics. At the outburst peak, the hot spot covers a large part $(\approx 50 \%)$ of the stellar surface. In quiescence, the hot spot is much smaller, i.e., $\lesssim 1 \%$ surface fraction. Similar results were determined for other eruptive YSOs (EX Lupi, Juhász et al. 2012, and V1118 Ori, Audard et al. 2010). Calvet \& Gullbring (1998) found fractions of $20 \%$ in strongly accreting systems, and typically $1 \%$ for T Tau systems. These surface fractions correspond to 6500 and $15000 \mathrm{~K}$ hot-spot temperatures at the outburst peak and quiescence, respectively.

\subsection{Dynamics of the inner disk and envelope}

During the outburst the temperatures were the highest at the peak, which defines the radius up to which dust had to evaporate. The variation of the inner radii of the disk and the envelope suggests that the physical mechanism enlarging the inner dustfree hole from 0.5 to $0.7 \mathrm{AU}$ is the evaporation of dust by the increased heating from the central source. The intensified wind from the center of the system might have also played a role in these changes (Reipurth \& Aspin 2004).

\subsection{Burst of luminosity or drop of extinction?}

Owing to the a structural change described previously, the extinction of the system should also change. The increased accretion luminosity and the varying extinction determine the total brightening of the object. When the heat because of the increased accretion luminosity evaporates dust particles and creates a larger inner hole, the line-of-sight extinction decreases, which leads to an additional apparent brightening of the object. Since the density in the envelope decreases with radius, most of the extinction comes from the innermost parts. Therefore, dust evaporation at the inner edge of the envelope can significantly change the total extinction toward the central star. Similarly, the gradual fading of the central source may allow recondensation of dust grains in regions where evaporation took place previously, resulting in an extra dimming. Kun et al. (2011) claimed that such re-condensation was observed at another YSO, PV Cep.
For the understanding of the outburst, it is essential to separate to which extent those two agents contributed to the brightening of V1647 Ori throughout the eruption. Some authors (e.g., McGehee et al. 2004; Reipurth \& Aspin 2004) concluded that only part of the brightness variation of V1647 Ori was due to extinction variation, e.g., dust-clearing. From our models, we derived a change of the extinction $\Delta A_{\mathrm{V}} \approx 4.5 \mathrm{mag}$ or $\Delta A_{\mathrm{I}_{\mathrm{C}}} \approx 2.5 \mathrm{mag}$. Compared to the largest measured variation in $I_{\mathrm{C}}$ (6.0 mag, AP07 and Aspin \& Reipurth 2009), it leaves more than 3 mag intrinsic brightening in $I_{C}$. There are a number of different attempts in the literature to derive the value of extinction and its variation during the outburst. Our model result for the quiescent phase $\left(A_{\mathrm{V}} \approx 23.4 \mathrm{mag}\right)$ agrees quite well with the value derived by Aspin et al. (2008, $\left.A_{\mathrm{V}} \approx 19 \mathrm{mag}\right)$. The object moved along the reddening path in the NIR color-color diagram during the outburst. The excursion corresponds to $\Delta A_{\mathrm{V}} \approx 5 \mathrm{mag}$ (e.g., Reipurth \& Aspin 2004), which is close to our results. However, with different methods, Aspin et al. (2008) derived $\Delta A_{\mathrm{V}} \approx 10 \mathrm{mag}$. Since the optical extinction values are poorly defined over the outburst, we do not aim at fitting them with our model, only attempt a qualitative check of this parameter. Our modeling indicated that the extinction is higher in quiescence than at peak brightness.

\subsection{Scenarios for variations seen by MIDI}

A blown-up spherical cavity The expected structural changes can either occur relatively slowly during the March-September interval or in a few weeks' time (cf. the light curves in Fig. 1). Even in the latter case such a change of the envelope seems to be plausible (corresponding to $100-200 \mathrm{~km} \mathrm{~s}^{-1}$ velocities). During most of this period the object was slowly fading, therefore we would expect the decrease of the inner (dust evaporation) radii both of the disk and the envelope rather than any increase. Combet \& Ferreira (2008) showed that at the accretion rate of $10^{-6} M_{\odot} / y r$, a strong disk wind, which can be the source of an outflow, is launched from the inner disk up to $2-3$ AU. Thus the disk wind might be responsible for the clearing of the inner envelope. However, during the 2005 March-September period the wind is expected to become weaker since its strength should be proportional to the accretion activity. Furthermore, the wind that was present from the beginning of the outburst and had no such effect before might only have cleared out the cone in the reflection nebula when the outburst started (Reipurth \& Aspin 2004). Therefore the stellar or disk wind is unlikely to have produced this clearing.

Another possibility is that some temporary outflow changed the inner structure of the system and moved the inner edge of the envelope outward. Spectroscopic observations from early 2006 showed evidence of a short outflow activity (Brittain et al. 2007). Furthermore, detection of forbidden optical lines indicated the presence of shocked gas in early 2006 (Fedele et al. 2007). These lines can be tracers of a Herbig-Haro object $(\mathrm{HHO})^{8}$. If such an event were responsible for the change of the inner radius of the envelope ( $\left.R_{\text {in,env }}\right)$, based on the beforementioned findings, it should be connected to the final decrease of the accretion rate. However, Brittain et al. (2007) proposed that because the inner radius of the accretion disk decreases together with the

\footnotetext{
8 Although HHOs are thought to be related to mass accretion, they are not typical in the environment of eruptive YSOs (HK96). Note that Eislöffel \& Mundt (1997) identified V1647 Ori as the driving source of $\mathrm{HH} 23$ - presumably ejected some thousands of years ago - which is located $155^{\prime \prime}$ north of the young star and is close to axis of the nebula.
} 
decreasing accretion rate, the re-structuring of the inner disk that produced the $(\mathrm{CO})$ outflow might have occured later.

During an outflow activity or the appearance of an HHO, the disk structure can be very different than that of a standard accretion disk. There is a significant difference in all parameters between those of a standard accretion disk and a jet-emitting disk (Combet \& Ferreira 2008). Reproducing such events are beyond the limits of our static modeling and can be a source of discrepancy.

By 2006 February, the object returned to its quiescent brightness at all wavelengths. This suggests that the structural changes in the circumstellar environment of V1647 Ori should have been reversible on the timescale of some months. It means that the material should fill the evacuated spherical cavity on this timescale via accretion, which would require an inward radial velocity of $10-20 \mathrm{~km} \mathrm{~s}^{-1}$. In our modeling it means that the inner radii of both the disk and the envelope should move back close to the quiescent values. Matter of the disk and the envelope might have moved inward due to the accretion and thus could fill the cleared regions. However, the dynamical timescale at $\sim 3 \mathrm{AU}$ is about seven years, much longer than half a year. Therefore dust recondensation might also have played a role here.

The disappearance of a warm halo. In the next scenario we again consider a large spherical inner cavity in the envelope as in Sect. $4.4\left(R_{\text {in,env }}=3.0 \mathrm{AU}\right.$ while $\left.R_{\text {in,disk }}=0.7 \mathrm{AU}\right)$, but we assume that the cavity has been produced at the early phases of the outburst for instance by stellar or disk winds (see e.g., Clarke et al. 2005). If dust was lifted from the disk above it by wind (Vinković \& Jurkić 2007; Sitko et al. 2008), the dust could fill the inner cavity of the envelope. Note that such a halo cannot be distinguished from the inner envelope in our models, but represent a different mechanism. The warm dust halo present in the system during the plateau phase makes V1647 Ori look more compact at the first MIDI epoch. The apparent change of the visibility data could then be caused by the waning wind. As the accretion process and thus the wind becomes weaker, less and less dust is fed into the cavity, while the dust moved there previously is blown farther outward. In this case most of the dust - which made the object compact in 2005 March - is cooled below the temperatures corresponding to the mid-infrared wavelengths. By 2005 September the cavity is practically cleared. Without carrying out new modeling, we can consider the results of the radiative transfer modeling described above. This scenario could explain the transition between 2005 March and September seen in the data and also the short timescale variability of the midinfrared emission.

An out-of-equilibrium system. Finally, we further speculate to find other alternatives for our series of static models. Between 2005 March and September the heated disk area should shrink gradually because of the fading of the central illuminating source. In contrast, the visibilities of V1647 Ori apparently did not follow such a scenario. The above hypothesis would predict a smaller - thus less resolved - source at our second MIDI epoch. Our measurements, however, revealed the opposite behavior: the visibilities decreased between the first and the second epochs. Since the shapes of the two visibility curves are similar (Fig. 1), and their ratio is almost independent of the wavelength $(\approx 1.4-1.5)$, a simple qualitative picture might explain the data. One could assume that the system consists of two components: one compact central and one extended component (e.g., approximated by a Gaussian brightness distribution as in Sect. 3.1). If the resolved component did not change between the two MIDI epochs, but the emission of the central unresolved source had significantly dropped in the same period, the emission of the system would have become less peaked, i.e., relatively more resolved. This might be a simplistic picture of what caused the decrease of the measured visibilities.

The constancy of the extended emission component could easily be explained if the $\sim 10 \mu \mathrm{m}$ flux arose from optically thick regions, whose temperature is not adjusted rapidly to the changing central illumination field. Although we see variations of the mid-IR brightness of the system on weekly timescales, that of an optically thick component could not change significantly because the corresponding timescales (Chiang \& Goldreich 1997) exceed even the difference between the epochs of the MIDI observations. However, according to Muzerolle et al. (2005), the contribution of an optically thick accretion disk to the midinfrared emission of V1647 Ori in outburst is small, and the $\sim 10 \mu$ m flux mainly arises from an envelope. In this envelope the temperature of the dust grains must be quickly adjusted to the external radiation field. This suggestion is supported by the fact that the mid-infrared flux of V1647 Ori had increased remarkably in less than a few months, between the beginning of the eruption and the Spitzer measurements in March 2004. Thus the invariability of the extended emitting component in the simple picture above is not straightforward to explain.

A qualitative argument leads to a possible solution in which the decrease seen in the MIDI visibilities is caused by the fading of the central source, which is not immediately followed by the fading of the outer regions. However, this effect should be confirmed by dynamical modeling and is beyond the scope of the present paper.

\section{Conclusions}

We performed interferometric and photometric observations of V1647 Ori during its 2003-2006 outburst to investigate the temporal evolution of its circumstellar structure and physical processes related to the eruption. In addition to the general fading of the object - shown by the multi-wavelength photometric data and archival mid-infrared spectroscopy, - short timescale variations were also observed. Optical-infrared SEDs at five epochs were compiled. Our radiative transfer modeling, with a smoothly decreasing accretion rate as a major varying parameter, provided good fits of the SEDs at different stages of the outburst. It is important to note that the inner radii of the dust disk and envelope also had to increase during the transition from quiescence to the outburst peak. The latter finding is clear evidence of dynamical variations in the inner circumstellar environment of V1647 Ori. This dust clearing is likely caused by the evaporation of the dust grains due to the outburst heat.

High angular resolution spatial information were also considered in the model-fitting procedure. VLTI/MIDI data obtained at two epochs, during both the slow and rapid fading stages, show a considerable change of the circumstellar structure. In constrast to our expectations, based on our model sequence, the object looked more resolved at the second epoch. One possible explanation can be a rapid removal of dust from the inner $3 \mathrm{AU}$ of the envelope, possibly caused by wind or outflow processes. In one case this spherical cavity is produced at the end of the outburst phase (the blown-up cavity scenario), or alternatively at the beginning (our warm dust halo scenario). Finally, we may also see V1647 Ori in a non-equilibrium situation in 2005 September, when the sudden fading of the central source was not yet followed by the fading of the optically thick circumstellar material. 
In general, our modeling showed that the circumstellar environment of V1647 Ori can be described by a disk and envelope system with parameters that are typical for embedded low-mass YSOs. This finding supports the hypothesis that eruptive YSOs are not peculiar objects, but represent an important phase in the evolution of all low-mass YSOs.

Acknowledgements. The authors thank the anonymous referee for his/her comments, which improved the manuscript. The authors thank the ESO/VLTI staff for executing the observations in service mode, Timea Csengeri for obtaining photometry at IAC, Aurora Sicilia-Aguilar and Kees Dullemond for useful discussion, Karl Gordon for the method of the MIPS data reduction. L.M. is grateful to Walter Jaffe and Rainer Köhler for helping with the MIDI data reduction. The research leading to these results has received funding from the European Community's Seventh Framework Programme under Grant Agreement 226604. L.M. and N.S. are thankful for the support of the Fizeau Exchange Visitor Program through the European Interferometry Initiative (EII) and OPTICON (an EU funded framework program, contract number RII3-CT-2004-001566). The research of Á.K. is supported by the Netherlands Organization for Scientific Research. Financial support from the Hungarian OTKA grants K81966, K101393 and NN102014 are acknowledged.

\section{Appendix A: Data reduction with different versions of MIA+EWS}

Since we aim at investigating temporal changes of the source structure, and the first MIDI datasets were reduced with the 1.3 version of MIA+EWS, we repeated the data reduction with the 1.6 version. It turned out that longward of $11 \mu \mathrm{m}$ the calibrated visibilities differ significantly with respect to the previous reduction, i.e., are higher than were shown in Paper I. At shorter wavelengths the results are consistent. Nonetheless, the results with EWS and MIA agreed well in Paper I. The EWS and MIA results are also consistent for the second epoch data (Sect. 2.1). As another test, we also reduced the second MIDI dataset with the older versions of the software. The results show a similar bias. In the case of MIA, the difference comes mainly from two changes mage since the first release. First, newer MIA versions use the photometry routine of EWS instead of its own (used in earlier versions). Second, these new versions of MIA fit fewer parameters of the mask than the older MIA version did. In the case of EWS, the difference is that its newer versions handle the data where the OPD difference is close to zero, differently from older EWS versions, i.e., these data are not considered anymore. Since all these changes of the softwares were made to improve the data reduction, we present calibrated visibility curves for both epochs produced wih MIA (1.6) and considered these for the radiative transfer modeling throughout the paper.

\section{References}

Ábrahám, P., Kóspál, Á., Csizmadia, Sz., et al. 2004, A\&A, 419, 39 Ábrahám, P., Mosoni, L., Henning, Th., et al. 2006, A\&A, 449, L13 Ábrahám, P., Juhász, A., Dullemond, C., et al. 2009, Nature, 459, 224 Acosta-Pulido, J. A., Kun, M., Ábrahám, P., et al. 2007, AJ, 133, 2020 Andrews, S. M., Rothberg, B., Simon, T. 2004, ApJ, 610, 45 Anthony-Twarog, B. J. 1982, AJ, 87, 1213 Aspin, C., \& Reipurth, B. 2009, AJ, 138, 1137
Aspin, C., Beck, T. L., \& Reipurth, B. 2008, AJ, 135, 423

Audard, M., Stringfellow, G. S., Güdel, M., et al. 2010, A\&A, 511, 63

Bell, K. R., Lin, D. N. C., Hartmann, L. W., \& Kenyon, S. J. 1995, ApJ, 444, 376

Beckwith, S. V. W. 1999, The Origin of Stars and Planetary Systems, eds. C. J. Lada, \& N. D. Kylafis (Kluwer Academic Publishers), 1999, 579

Bonnell, I., \& Bastien, P. 1992, ApJ, 401, 31

Briceño, C., Vivas, A. K., Hernandez, J., et al. 2004, ApJ, 606, 123

Brittain, S., Rettig, T. W., Simon, T., et al. 2007, ApJ, 670, 29

Calvet, N., \& Gullbring, E. 1998, ApJ, 509, 802

Chesneau, O. 2007, NewAR, 51, 666

Chiang, E. I., \& Goldreich, P. 1997, ApJ, 490, 368

Clarke, C., Lodato, G., Melnikov, S. Y., \& Ibrahimov, M. A. 2005, MNRAS, 361,942

Combet, C., \& Ferreira, J. 2008, A\&A, 479, 481

Dale, D. A., Cohen, S. A., Johnson, L. C., et al. 2009, ApJ, 703, 517

D’Angelo, C., \& Spruit, H. C. 2010, MNRAS, 406, 1208

Eislöffel, J., \& Mundt, R. 1997, AJ, 114, 280

Eisner, J. A., \& Hillenbrand, L. A. 2011, ApJ, 738, 9

Evans, N. J. II, Dunham, M. M., Jørgensen, J. K., et al. 2009, ApJS, 181, 321

Fedele, D., van den Ancker, M. E., Petr-Gotzens, M. G., \& Rafanelli, P. 2007, A\&A, 472, 207

Gordon, K. D., Engelbracht, C. W., Fadda, D., et al. 2007, PASP, 119, 1019

Gullbring, E., Hartmann, L. W., Briceño, C., \& Calvet, N. 1998, ApJ, 492, 323

Hartmann, L. W., \& Kenyon, S. J. 1996, ARA\&A, 34, 207

Hartmann, L., Calvet, N., Gullbring, E., \& D’Alessio, P. 1998, ApJ, 495, 385

Herbig, G. H. 2007, AJ, 133, 2679

Herbst, W., Herbst, D. K., Grossman, E. J., et al. 1994, AJ, 108, 1906

Hora, J. L., Carey, S., Surace, J., et al. 2008, PASP, 120, 1233

Juhász, A., Dullemond, C., van Boekel, R., et al. 2012, ApJ, 744, 118

Kun, M. Szegedi-Elek, E., Moór, A., et al. 2011, MNRAS, 413, 2689

Leinert, Ch., Graser, U., Richichi, A., et al. 2003, ESO Messenger, 112, 13

Leinert, Ch., van Boekel, R., Waters, L. B. F. M., et al. 2004, A\&A, 423, 537

Lis, D. C., Menten, K. M., \& Zylka, R. 1999, ApJ, 527, 856

Lodato, G., \& Clarke, C. 2004, MNRAS, 353, 841

Lu, N., Smith, P. S., Engelbracht, C. W., et al. 2008, PASP, 120, 328

Makovoz, D., \& Marleau, F. R. 2005, PASP, 117, 1113

McGehee, P. M., Smith, J. A., Henden, A. A., et al. 2004, ApJ, 616, 1058

McNeil, J. W. 2004, IAU Circ., 8284, 1

Muzerolle, J., Megeath, S. T., Flaherty, K. M., et al. 2005, ApJ, 620, 107

Natta, A. 1993, ApJ, 412, 761

Quanz, S. P., Henning, Th., Bouwman, J., et al. 2006, ApJ, 648, 472

Quanz, S. P., Henning, Th., Bouwman, J., et al. 2007, ApJ, 668, 359

Ratzka, Th., Leinert, Ch., Henning, Th., et al. 2007, A\&A, 471, 173

Ratzka, Th., Schegerer, A. A., Leinert, Ch., et al. 2009, A\&A, 502, 623

Reipurth, B., \& Aspin, C. 2004, ApJ, 606, 119

Rettig, T. W., Brittain, S. D., Gibb, E. L., et al. 2005, ApJ, 626, 24

Rieke, G. H., Young, E. T., Engelbracht, C. W., et al. 2004, ApJS, 154, 25

Sandell, G., \& Weintraub, D. A. 2001, ApJS, 134, 115

Schegerer, A. A., Wolf, S., Ratzka, Th., \& Leinert, Ch. 2008, A\&A, 478, 779

Sicilia-Aguilar, A., Merin, B., Hormuth, F., et al. 2008, ApJ, 673, 382

Sipos, N., Ábrahám, P., Acosta-Pulido, J., et al. 2009, A\&A, 507, 881

Sitko, M. L., Carpenter, W. J., Kimes, R. L., et al. 2008, ApJ, 678, 1070

Shu, F. H. 1977, ApJ, 214, 488

Teets, W. K., Weintraub, D. A., Grosso, N., et al. 2001, ApJ, 741, 83

Tsukagoshi, T., Kitamura, Y., \& Kawabe, R. 2005, PASJ, 57, 21

Vacca, W. D., Cushing, M. C., \& Simon, T. 2004, ApJ, 609, 29

van Boekel, R., Min, M., Leinert, Ch., et al. 2004, Nature, 432, 479

van Boekel, R., Juhász, A., Henning, Th., et al. 2010, A\&A, 517, A16

Vorobyov, E. I., \& Basu, S. 2006, ApJ, 650, 956

Vinković, D., \& Jurkić, T. 2007, ApJ, 658, 462

Weingartner, J. C., \& Draine, B. T. 2001, ApJ, 548, 296

White, R. J., \& Hillenbrand, L. A. 2004, ApJ, 616, 998

Whitney, B., Wood, K., Bjorkman, J. E., \& Wolff, M. J. 2003, ApJ, 591, 1049

Wolf, S. 2003, Comput. Phys. Comm., 150, 99

Wolf, S., Henning, Th., \& Stecklum, B. 1999, A\&A, 349, 839

Wood, K., Lada, C. J., Bjorkman, J. E., et al. 2002, ApJ, 567, 1183

Zhu, Z., Hartmann, L., Gammie, C., \& McKinney, J. C. 2009, ApJ, 701, 620 